\title{
Conformal invariance of TMD rapidity evolution
}

\author{
Giovanni Antonio Chirilli* \\ Institut für Theoretische Physik, Universität Regensburg, \\ D-93040 Regensburg, Germany \\ E-mail: giovanni.chirillieur.de
}

\begin{abstract}
Very well known schemes to regulate the rapidity/UV divergences of the Transverse Momentum Distribution operators due to the infinite light-like gauge links are the Collis Soper Sterman formalism or the Soft Collinear Effective Theory formalism. An alternative choice is provided by the scheme used in the small-x physics. The corresponding evolution equations differ already at leading order. In view of the future Electron Ion Collider accelerator, which will probe the TMDs at values of the Bjorken $\mathrm{x}$ in the region between small- $\mathrm{x}$ to $\mathrm{x} \sim 1$, the different formalisms need to be reconciled. I will discuss the conformal properties of TMD operators and present the result of the conformal rapidity evolution of TMD operators in the Sudakov region.
\end{abstract}

Light Cone 2019 - QCD on the light cone: from hadrons to heavy ions - LC2019

16-20 September 2019

Ecole Polytechnique, Palaiseau, France

*Speaker. 


\section{Introduction}

The TMDs (for a review, see Ref. [1]) are defined as matrix elements of quark or gluon operators with attached light-like gauge links (Wilson lines) going to either $+\infty$ or $-\infty$ depending on the process under consideration. It is well known that these TMD operators exhibit rapidity divergences due to infinite light-like gauge links and the corresponding rapidity/UV divergences should be regularized. Known schemes are based on CSS or SCET formalism. An alternative scheme is inspired by the small- $x$ physics $[2,3]$. The obtained evolution equations differ even at the leading-order level and need to be reconciled, especially in view of the future EIC accelerator which will probe the TMDs at values of Bjorken $x$ between small- $x$ and $x \sim 1$ regions.

A good starting point is to obtain conformal leading-order evolution equations. In our case, since TMD operators are defined with attached light-like Wilson lines, formally they will transform covariantly under the subgroup of full conformal group which preserves this light-like direction. However, as we mentioned, the TMD operators contain rapidity divergencies which need to be regularized. At present, there is no rapidity cutoff which preserves conformal invariance so the best one can do is to find the cutoff which is conformal at the leading order in perturbation theory. In higher orders, one should not expect conformal invariance since it is broken by running of QCD coupling. However, if one considers corresponding correlation functions in $\mathscr{N}=4 \mathrm{SYM}$, one should expect conformal invariance. After that, the results obtained in $\mathscr{N}=4 \mathrm{SYM}$ theory can be used as a starting point of QCD calculation. Thus, the idea is to find TMD operator conformal in $\mathscr{N}=4 \mathrm{SYM}$ and use it in QCD.

In the next sections we report results published in Ref. [7].

\section{Conformal invariance of TMD operators}

For simplicity, we will consider the gluon operators with light-like Wilson lines stretching to $-\infty$ in "+" direction. The gluon TMD (unintegrated gluon distribution) is defined as [4]

$$
\begin{aligned}
& \mathscr{D}\left(x_{B}, k_{\perp}, \eta\right)=\int d^{2} z_{\perp} e^{i(k, z) \perp} \mathscr{D}\left(x_{B}, z_{\perp}, \eta\right), \\
& g^{2} \mathscr{D}\left(x_{B}, z_{\perp}, \eta\right) \stackrel{z_{-}=0}{=} \frac{-x_{B}^{-1}}{2 \pi p^{-}} \int d z^{+} e^{-i x_{B} p^{-} z^{+}}\left\langle P\left|\mathscr{F}_{\xi}^{a}(z)[z-\infty n,-\infty n]^{a b} \mathscr{F}^{b \xi}(0)\right| P\right\rangle
\end{aligned}
$$

where $|P\rangle$ is an unpolarized target with momentum $p \simeq p^{-}$(typically proton) and $n=\left(\frac{1}{\sqrt{2}}, 0,0, \frac{1}{\sqrt{2}}\right)$ is a light-like vector in "+" direction. Hereafter we use the notation

$$
\left.\mathscr{F}^{\xi, a}\left(z_{\perp}, z^{+}\right) \equiv g F^{-\xi, m}(z)[z, z-\infty n]^{m a}\right|_{z^{-}=0}
$$

where $[x, y]$ denotes straight-line gauge link connecting points $x$ and $y:[x, y] \equiv \mathrm{P} e^{i g \int d u(x-y)^{\mu} A_{\mu}(u x+(1-u) y)}$ To simplify one-loop evolution we multiplied $F_{\mu \nu}$ by coupling constant. Since the $g A_{\mu}$ is renorminvariant we do not need to consider self-energy diagrams (in the background-Feynman gauge). Note that $z^{-}=0$ is fixed by the original factorization formula for particle production [1] (see also the discussion in Ref. [5, 6]).

The algebra of full conformal group $S O(2,4)$ consists of four operators $P^{\mu}$, six $M^{\mu \nu}$, four special conformal generators $K^{\mu}$, and dilatation operator $D$. It is easy to check that in the leading 
order the following 11 operators act on gluon TMDs covariantly [7]

$$
P^{i}, P^{-}, M^{12}, M^{-i}, D, K^{i}, K^{-}, M^{-+}
$$

while the action of operators $P^{+}, M^{+i}$, and $K^{+}$do not preserve the form of the operator (2.2). The corresponding group consists of transformations which leave the hyperplane $z^{-}=0$ and vector $n$ invariant. Those include shifts in transverse and " + " directions, rotations in the transverse plane, Lorentz rotations/boosts created by $M^{-i}$, dilatations, and special conformal transformations $z_{\mu}^{\prime}=\frac{z_{\mu}-a_{\mu} z^{2}}{1-2 a \cdot z+a^{2} z^{2}}$ with $a=\left(a^{+}, 0, a_{\perp}\right)$.

As we noted, infinite Wilson lines in the definition (2.2) of TMD operators make them divergent. As we discussed above, it is very advantageous to have a cutoff of these divergencies compatible with approximate conformal invariance of tree-level QCD. The evolution equation with such cutoff should be invariant with respect to transformations described above.

\section{TMD factorization and one-loop evolution in the Sudakov region}

It is convenient to consider as a starting point the simple case of TMD evolution in the socalled Sudakov region corresponding to small longitudinal distances. First, let us specify what we call a Sudakov region. A typical factorization formula for the differential cross section of particle production in hadron-hadron collision is $[1,8]$

$$
\frac{d \sigma}{d \eta d^{2} q_{\perp}}=\sum_{f} \int d^{2} b_{\perp} e^{i(q, b)_{\perp}} \mathscr{D}_{f / A}\left(x_{A}, b_{\perp}, \eta\right) \mathscr{D}_{f / B}\left(x_{B}, b_{\perp}, \eta\right) \sigma(f f \rightarrow H)+\ldots
$$

where $\eta=\frac{1}{2} \ln \frac{q^{+}}{q^{-}}$is the rapidity, $\mathscr{D}_{f / h}\left(x, z_{\perp}, \eta\right)$ is the TMD density of a parton $f$ in hadron $h$, and $\sigma(f f \rightarrow H)$ is the cross section of production of particle $H$ of invariant mass $m_{H}^{2}=q^{2} \equiv Q^{2}$ in the scattering of two partons. (One can keep in mind Higgs production in the approximation of point-like gluon-gluon-Higgs vertex). The Sudakov region is defined by $Q \gg q_{\perp} \gg 1 \mathrm{GeV}$ since at such kinematics there is a double-log evolution for transverse momenta between $Q$ and $q_{\perp}$. In the coordinate space, TMD factorization (3.1) looks like

$$
\begin{aligned}
& \left\langle p_{A}, p_{B}\left|g^{2} F_{\mu v}^{a} F^{a \mu v}\left(z_{1}\right) g^{2} F_{\lambda \rho}^{b} F^{b \lambda \rho}\left(z_{2}\right)\right| p_{A}, p_{B}\right\rangle \\
= & \frac{1}{N_{c}^{2}-1}\left\langle p_{A}\left|\tilde{\mathscr{O}}_{i j}\left(z_{1}^{-}, z_{1_{\perp}} ; z_{2}^{-}, z_{2_{\perp}}\right)\right| p_{A}\right\rangle^{\sigma_{A}}\left\langle p_{B}\left|\mathscr{O}^{i j}\left(z_{1}^{+}, z_{1_{\perp}} ; z_{2}^{+}, z_{2_{\perp}}\right)\right| p_{B}\right\rangle^{\sigma_{B}}+\ldots
\end{aligned}
$$

where $\mathscr{O}_{i j}, \tilde{\mathscr{O}}_{i j}$, and $\mathscr{F}^{i, a}$ are defined in Ref. [7]. Here $p_{A}=\sqrt{\frac{s}{2}} n+\frac{p_{A}^{2}}{\sqrt{2 s}} n^{\prime}, p_{B}=\sqrt{\frac{2}{s}} n^{\prime}+\frac{p_{B}^{2}}{\sqrt{2 s}} n$ and $n^{\prime}=\left(\frac{1}{\sqrt{2}}, 0,0,-\frac{1}{\sqrt{2}}\right)$. Our metric is $x^{2}=2 x^{+} x^{-}-x_{\perp}^{2}$.

As we mentioned, TMD operators exhibit rapidity divergencies due to infinite light-like gauge links. The "small- $x$ style" rapidity cutoff for longitudinal divergencies is imposed as the upper limit of $k^{+}$components of gluons emitted from the Wilson lines. As we will see below, to get the conformal invariance of the leading-order evolution we need to impose the cutoff of $k^{+}$components of gluons correlated with transverse size of TMD in the following way:

$$
\begin{aligned}
& \mathscr{F}^{i, a}\left(z_{\perp}, z^{+}\right)^{\sigma} \equiv g F^{-i, m}(z)\left[\mathrm{P} e^{i g \int_{-\infty}^{+} d z^{+} A^{-, \sigma}\left(u p_{1}+x_{\perp}\right)}\right]^{m a}, \\
& A_{\mu}^{\sigma}(x)=\int \frac{d^{4} k}{16 \pi^{4}} \theta\left(\frac{\sigma \sqrt{2}}{z_{12_{\perp}}}-\left|k^{+}\right|\right) e^{-i k \cdot x} A_{\mu}(k)
\end{aligned}
$$


Similarly, the operator $\tilde{\mathscr{O}}$ is defined with with the rapidity cutoff for $\beta$ integration imposed as $\theta\left(\frac{\tilde{\sigma} \sqrt{2}}{z_{12}}-\left|k^{-}\right|\right)$.
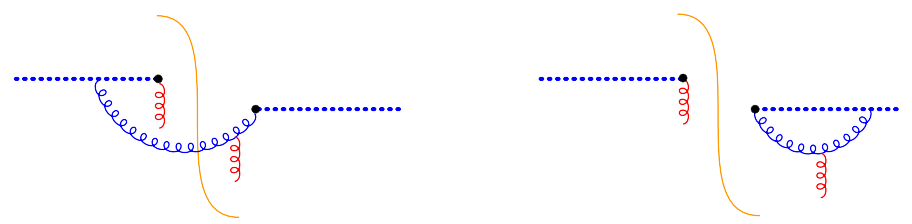

Figure 1: Typical diagrams for production (a) and virtual (b) contributions to the evolution kernel. The dashed lines denote gauge links.

The Sudakov region $Q^{2} \gg q_{\perp}^{2}$ in the coordinate space corresponds to $z_{12}^{2} \equiv 2 z_{12}^{-} z_{12}^{+} \ll z_{12}^{2}$ where $z_{12} \equiv z_{1}-z_{2}$. In the leading log approximation, the upper cutoff for $k^{+}$integration in the target matrix element in Eq. (3.2) is $\sigma_{B}=\frac{1}{\sqrt{2}} \frac{z_{12} \downarrow}{z_{12}^{-}}$and similarly the $\beta$-integration cutoff in projectile matrix element is $\sigma_{A}=\frac{1}{\sqrt{2}} \frac{z_{12_{\perp}}}{z_{12}^{+}}$.

Let us derive the evolution of gluon TMD operator with respect to cutoff $\sigma$ in the leading log approximation. As usual, to get an evolution equation we integrate over momenta $\frac{\sigma_{2} \sqrt{2}}{z_{12}}>k^{+}>$ $\frac{\sigma_{1} \sqrt{2}}{z_{12}}$. To this end, we calculate diagrams shown in Fig. 1 in the background field of gluons with $k^{+}<\frac{\sigma_{1} \sqrt{2}}{z_{12}}$. The result is

$$
\mathscr{O}^{\sigma_{2}}\left(z_{1}^{+}, z_{2}^{+}\right)=\frac{\alpha_{s} N_{c}}{2 \pi} \int_{\frac{\sigma_{1} \sqrt{2}}{z_{12}}}^{\frac{\sigma_{2} \sqrt{2}}{z_{12}}} \frac{d k^{+}}{k^{+}} K \mathscr{O}^{\sigma_{1}}\left(z_{1}^{+}, z_{2}^{+}\right)
$$

where the kernel $K$ is given by [7]

$$
\begin{aligned}
& K \mathscr{O}\left(z_{1}^{+}, z_{2}^{+}\right)=\mathscr{O}\left(z_{1}^{+}, z_{2}^{+}\right) \int_{-\infty}^{z_{1}^{+}} \frac{d z^{+}}{z_{2}^{+}-z^{+}} e^{-i \frac{z_{12}, \sigma}{\sqrt{2}\left(z_{2}-z\right)^{+}}}+\mathscr{O}\left(z_{1}^{+}, z_{2}^{+}\right) \int_{-\infty}^{z_{2}^{+}} \frac{d z^{+}}{z_{1}^{+}-z^{+}} e^{i \frac{z_{12}, \sigma}{\sqrt{2}\left(z_{1}-z\right)^{+}}} \\
& -\int_{-\infty}^{z_{1}^{+}} d z^{+} \frac{\mathscr{O}\left(z_{1}^{+}, z_{2}^{+}\right)-\mathscr{O}\left(z^{+}, z_{2}^{+}\right)}{z_{1}^{+}-z^{+}}-\int_{-\infty}^{z_{2}^{+}} d z^{+} \frac{\mathscr{O}\left(z_{1}^{+}, z_{2}^{+}\right)-\mathscr{O}\left(z_{1}^{+}, z^{+}\right)}{z_{2}^{+}-z^{+}}
\end{aligned}
$$

where we suppress arguments $z_{1_{\perp}}$ and $z_{2_{\perp}}$ since they do not change during the evolution in the Sudakov regime. The first two terms in the kernel $K$ come from the "production" diagram in Fig. 1a while the last two terms from "virtual" diagram in Fig. 1b. The approximations for diagrams in Fig. 1 leading to Eq. (3.5) are valid as long as $k^{+} \gg \frac{z_{12}^{+}}{z_{12}^{2}}$ which gives the region of applicability of Sudakov-type evolution.

Evolution equation (3.4) can be easily integrated using Fourier transformation (see [7] for details) and one easily obtains

$$
\begin{aligned}
& \mathscr{O}^{\sigma_{2}}\left(z_{1}^{+}, z_{2}^{+}\right)=e^{-2 \bar{\alpha}_{s} \ln \frac{\sigma_{2}}{\sigma_{1}}\left[\ln \sigma_{1} \sigma_{2}+4 \gamma_{E}-\ln 2\right]} \int d z_{1}^{\prime+} d z_{2}^{\prime+} \mathscr{O}^{\sigma_{1}}\left(z_{1}^{\prime+}, z_{2}^{\prime+}\right) z_{12_{\perp}}^{-2 \bar{\alpha}_{s} \ln \frac{\sigma_{2}}{\sigma_{1}}} \\
& \times \frac{1}{4 \pi^{2}}\left[\frac{i \Gamma\left(1-2 \bar{\alpha}_{s} \ln \frac{\sigma_{2}}{\sigma_{1}}\right)}{\left(z_{1}^{+}-z_{1}^{\prime+}+i \varepsilon\right)^{1-2 \bar{\alpha}_{s} \ln \frac{\sigma_{2}}{\sigma_{1}}}}+c . c .\right]\left[\frac{i \Gamma\left(1-2 \bar{\alpha}_{s} \ln \frac{\sigma_{2}}{\sigma_{1}}\right)}{\left(z_{2}^{+}-z_{2}^{\prime+}+i \varepsilon\right)^{1-2 \bar{\alpha}_{s} \ln \frac{\sigma_{2}}{\sigma_{1}}}+c . c .}\right]
\end{aligned}
$$

\footnotetext{
${ }^{1}$ Hereafter we use the simplified notation $z_{12} \equiv\left|z_{12_{\perp}}\right|$.
} 
where we introduced notation $\bar{\alpha}_{s} \equiv \frac{\alpha_{s} N_{c}}{4 \pi}$. It should be mentioned that the factor $4 \gamma_{E}$ is "schemedependent": if one introduces to $\alpha$-integrals smooth cutoff $e^{-\alpha / a}$ instead of rigid cutoff $\theta(a>\alpha)$, the value $4 \gamma_{E}$ changes to $2 \gamma_{E}$. It is easy to see that the r.h.s. of Eq. (3.6) transforms covariantly under all transformations (2.3) except Lorentz boost generated by $M^{+-}$(see [7] for details).

\section{Conclusions and Outlook}

The first result is that the 11-parameter subgroup of $S O(2,4)$ formed by generators (2.3) formally leaves TMD operators invariant.

The second result is related to the fact that conformal invariance is violated by the rapidity cutoff (even in $\mathscr{N}=4 \mathrm{SYM}$ ). We have studied the TMD evolution in the Sudakov region of intermediate $x$ and demonstrated that the rapidity cutoff used in small- $x$ literature preserves all generators of our subgroup except the Lorentz boost which is related to the change of that cutoff.

Our main outlook is to try to connect to small- $x$ region, first in $\mathscr{N}=4 \mathrm{SYM}$ and then in QCD. As we mentioned above, although the TMD evolution in a small $x$ region is conformal with respect to $S L(2, C)$ group, and our evolution (3.6) is also conformal (albeit with respect to different group of which $S L(2, C)$ is a subgroup), the transition between Sudakov region and small- $x$ region is described by rather complicated interpolation formula [9] which is not conformally invariant. Our hope is that in a conformal theory one can simplify that transition using the conformal invariance requirement. The study is in progress.

\section{References}

[1] J. Collins, Foundations of perturbative QCD (Cambridge University Press, 2013), ISBN isbn: 9781107645257, 9781107645257, 9780521855334,9781139097826, URL http://www. cambridge.org/de/knowledge/isbn/item5756723.

[2] L. N. Lipatov, Phys. Rept. 286 (1997) 131 doi:10.1016/S0370-1573(96)00045-2 [hep-ph/9610276].

[3] Y. V. Kovchegov and E. Levin, Camb. Monogr. Part. Phys. Nucl. Phys. Cosmol. 33 (2012) 1. doi:10.1017/CBO9781139022187

[4] P. J. Mulders and J. Rodrigues, Phys. Rev. D 63 (2001) 094021 doi:10.1103/PhysRevD.63.094021 [hep-ph/0009343].

[5] I. Balitsky and A. Tarasov, JHEP 1707 (2017) 095 doi:10.1007/JHEP07(2017)095 [arXiv:1706.01415 [hep-ph]].

[6] I. Balitsky and A. Tarasov, JHEP 1805 (2018) 150 doi:10.1007/JHEP05(2018)150 [arXiv:1712.09389 [hep-ph]].

[7] I. Balitsky and G. A. Chirilli, Phys. Rev. D 100 (2019) no.5, 051504 doi:10.1103/PhysRevD.100.051504 [arXiv:1905.09144 [hep-ph]].

[8] J. Collins and T. Rogers, Phys. Rev. D 91 (2015) no.7, 074020 doi:10.1103/PhysRevD.91.074020 [arXiv:1412.3820 [hep-ph]].

[9] I. Balitsky and A. Tarasov, JHEP 1510 (2015) 017 doi:10.1007/JHEP10(2015)017 [arXiv:1505.02151 [hep-ph]]. 